\title{
A Novel Process Network Model for Interacting Context-aware Web Services
}

\author{
Xiuguo Zhang, Hongbo Liu, Ajith Abraham Senior Member, IEEE
}

\begin{abstract}
Context-aware web services have been attracting significant attention as an important approach for improving the usability of web services. In this paper, we explore a novel approach to model dynamic behaviors of interacting context-aware web services, aiming to effectively process and take advantage of contexts and realize behavior adaptation of web services and further to facilitate the development of context-aware application of web services. We present an interaction model of context-aware web services based on Context-aware Process Network (CAPN), which is a dataflow and channel based model of cooperative computation. CAPN is extended to context-aware web service network by introducing a kind of sensor processes, which is used to catch contextual data from external environment. Through modeling the register link's behaviors, we present how a web service can respond to its context changes dynamically. The formal behavior semantics of our model is described by Calculus of Communicating Systems (CCS) process algebra. The behavior adaptation and context-awareness in our model are discussed. A XML (eXtensible Markup Language) formatted service behavior description language named BML4WS is designed to describe behaviors and behavior adaptation of interacting context-aware web services. Finally, an application case is demonstrated to illustrate the proposed model how to adapt context changes and describe service behaviours and their changes.
\end{abstract}

Index Terms-Context-aware web service, service behavior, CCS process algebra, CAPN, service interaction.

\section{INTRODUCTION}

C Ontext-aware web service can help its system to understand situational context and share that context with other services. "Context" mainly refers to the information about clients and their environment that may be used by web services to provide clients with a customized and personalized behavior [1], [2]. Context information includes any additional information, e.g. a consumer's name, current location and address, type of client device and so on, which can be used to improve the behavior of a service in a situation [3], [4], [5], [6]. Without such additional information, the service would be operable as normal but with context information, which is arguable that the service can operate better or more appropriately [Z], [8], [9], [10]. Context-awareness is considered as a kind of ability of the application to discover and take advantage of context information. In context-aware application of web services [11], [12], a web service can adapt its operations according to its contexts, that is, service behaviors change as the context changes. In order to respond to context changes, a contextaware web service has to accommodate for a variety of context types and tune their behaviors dynamically with the changing contexts. Context-aware web services have been attracting significant attention as an

- X. Zhang and H. Liu are with the School of Information Science and Technology at Dalian Maritime University, Dalian, 116026. E-mail: $\{$ zhangxg,lhb\}@dlmu.edu.cn

- A. Abraham is a Director at Machine Intelligence Research Labs, Seattle, Washington 98071, USA.

E-mail: ajith.abraham@ieee.org important approach to improving the usability of web services [13], [14], [15], [16], [17], [18], [19].

In this paper, we focus on the behavior of interacting context-aware web services and automatic behavior adaptation of web services to context changes. We tackle the problem of how contexts are processed and how they affect service behaviors dynamically. A novel approach is proposed to model dynamic behaviors of interacting context-aware web services, aiming to effectively process and take advantage of contexts and realize behavior adaptation of web services and further to facilitate the development of context-aware application of web services. We present an interaction model of context-aware web services based on a formal model, Context-aware Process Network (CAPN) [20], [2T], which is a dataflow and channel based model of cooperative computation. CAPN is extended to context-aware web service network by adding a kind of additional sensor processes which is used to catch contextual data from external environment. In CAPN, the application data and context data are not distinctly separated, while in context-aware web service network, the separation of application data and context data is enforced by separating respective reading and writing semantics. This separation helps us to realize behavior adaptation of context-aware web service. We use unidirectional register link of CAPN to carry context information of web services. Through modeling the register link's behaviors, we present how the web service's behavior dynamically changes as context changes. Using CAPN, we aim to reduce unanticipated circumstances and uncooperative cases of interacting web services. 
This paper is organized as follows: Related works are presented in Section 2 . Section B describes the service interaction model of context-aware web service network. Section $\mathbb{4}$ presents behavior semantics of context-aware web service network. Section $\square$ introduces the realization of behavior adaptation and context-awareness in context-aware web service network. Section $\mathbf{6}$ provides a XML formatted service behavior description language for describing behaviors and behavior adaptation of interacting contextaware web services. Section $\square$ presents a case to show how the application can adapt to context changes and how to describe service behaviours and their changes. Section 8 draws conclusions and future work.

mds

May 1, 2011

\section{Related Works}

\subsection{Context-aware Web Service}

Recently, different technologies, approaches and frameworks for context-aware web services and their application have been researched [1] [ [4], [22], [2.3], [24], [25], [26], [27]. A context-aware web service is a smart web service, which can understand situational context and can share that context with other services [4], [28]. In this paper, we are mainly concerned about dynamic behaviors of interacting web services. We consider a web service as context-aware if it uses context to provide customized and personalized behaviors during its interaction with other services.

The definition of context depends on the purpose of the application. For example, in its broad sense in [29], context is defined as any information that can be used to characterize the situation of an entity. An entity is a person, place or object that is considered relevant or the interaction between a user and an application, including the user and applications themselves. For web services [5], context is defined as the information characterizing the situation in which the services are being executed. In this paper, we consider a context as any information that can be used to characterize the situation of interactions among web services, including state of interaction environment, state of entities in interaction environment such as user state and web service state.

Context types also differ form situation to situation. For example, Keidl et al. [1], presented several context types including the consumer's location, client's devices, information about the consumer invoking the web service, e.g., name and email address, connection preferences, which allows to specify properties of the connections to Web services. In [29], three categories of context are distinguished: physical (location, time, etc), computing (terminal form-factor, battery life, available bandwidth, accessible computing facilities, etc) and user context (current activity, schedule, intent, etc). In this paper, we use three kinds of contexts to characterize the situation of service interactions, including interaction environment context (network bandwidth, operating system, client's devices, etc), user context (user preference, user location, etc) and service context (business restriction, service availability, execution time, success rate, etc).

\subsection{Web Service Behavior Modeling}

At present, the main methods of modeling web service behaviors are Petri Net based method [30], [3T], process algebra based method [32], [33] and finite state machine based method [34]. These methods are formal and commonly used to describe and verify web service behaviors. For example, Petri Net based behavior modeling method focus on describing behavior process inside web service and is fit to verify correctness of composite service behaviors. Process algebra based behavior modeling method focus on describing behavior process outside web service and is fit to verify interaction correctness among web services. Finite state machine based method describes service behaviors from the view of state transition and message passing. But it is difficult to describe behaviors of sophisticated process using finite state machine.

In this paper, we use Context-aware Process Network (CAPN) to model dynamic behaviors of interacting context-aware web services. We think CAPN is more fit for modeling interacting context-aware web services in several aspects, including its parallelism and communication mechanism, compositional property, executable property and context-awareness semantics.

\subsection{Behavior Adaptation of Context-aware Web Services}

Adaptation is the capability to provide different versions of a service or different presentations of a document, in order to suite the needs of the user, of the environment, of the equipment, etc [35]. In this paper, we consider behavior adaptation of a contextaware web service as its capability to provide different versions of customized and personalized behaviors for meeting the needs of context changes.

Chaari et al. [36] categorized context adaptation into three categories, which are services adaptation, content adaptation and UI adaptation. Here, services refer to the services of the application, content is the exchanged data with the user and UI is the visualization. They developed a platform which makes the services, data and the user interface of applications adaptable to different context situations.

Jacob et al. [25] defined the notion of context functions which is a statement about the relative influence of a context parameter on the behavior of the respective service. Context functions can be a mathematical function or a regular expression and can be easily 
adapted to directly affect the context-aware service behavior.

Zhou et al. [26] proposed the concept of ContextAware Pervasive Service Composition (CAPSC) and design a CAPSC architecture by taking into account context-aware peer coordination, context-aware process service adaptation, and context-aware utility service adaptation.

Hervas et al. [27] exploited semantics to apply context in run-time adaptation. They implement mechanisms to support the dynamic behavior of the users and their surroundings, including techniques to adapt context model to their future needs, to maintain the context information at run-time and to be interoperable with external context models.

To summarize, different behavior adaptation approaches tackle the problem of behavior adaptation in different manners. In this paper, based on CAPN model, we present our own context processing and behavior adaptation approaches. Similar to services adaptation in [36], we also provide different behavior versions for a context-aware web service, but we provide a register link based behavior adaptation strategy and a more detailed version selection approach. We adopt context influence functions to denote the influence of contexts on web service. The influence function in this work is similar to context function in [25], but their context function is not used for behavior version selection of context-aware web service. Also our behavior adaptation strategy is different from [25], since we provide a unified and formatted influence function for every context in order to calculate the rank of a behavior version, but [25] does not. Using CAPN to model context-aware web service interaction, we can present an explicit formal semantic for interacting context-aware web service that facilitates the development of context-aware application of web services.

\section{Context-aware Web Service Net- WORK: A SERVICE INTERACTION MODEL}

Service interaction describes how services can communicate with each other at the message level. In this section, we describe an interaction model of contextaware web services. We name this interaction model as a context-aware web service network. Its design idea comes from a dataflow and channel based model of cooperative computation, i.e. Context-aware Process Network [20], [2T]. Next, we briefly introduce the model of context-aware process network.

\subsection{Context-aware Process Network}

Kahn Process Network (KPN) [20] is a model of computation based on dataflow and has been widely used in many areas [37], [38], [39]. A strong aspect of KPNs is that they make (task-level) parallelism and communication in an application explicit, which means that they are very suitable for execution on distributed architectures. In KPN, nodes represent computations (concurrent processes) and arcs represent totally ordered sequences of data (commonly called tokens). Concurrent processes communicate with each other using FIFO channels which are unidirectional unbounded queues of data tokens between two processes. Read actions from these FIFOs block until at least one data item (or token) becomes available. The FIFOs have unbounded size, so writing actions are non-blocking. Reading actions from the FIFOs are destructive, which means that a token can only be read once. KPN are determinate, i.e. given an input, the output result of its computation is independent of execution order. This allows us to execute the network in parallel, as well as sequentially. KPNs implement asynchronous communication but synchronous coordination. Geilen and Basten [40] presented an operational semantics for Kahn Process Network.

In order to facilitate asynchronous coordination, H. van Dijk et al. [2T] extended the Kahn model of computations to Context-aware Process Network (CAPN) with a simple indeterminate construct, i.e. a unidirectional non-blocking register link (REG), which has destructive and replicative behavior. They defined the behavior of a REG link as follows. Writing to a full register overwrites a previously written value. Reading from a register returns the last received value; a value can be returned multiple times. Because the context information in REG as control information may decide the output of a process, i.e. given an input, the output result of its computation changes as contexts change, so CAPN is indeterminate.

\subsection{Context-aware Web Service Network}

Several advantages of CAPN make it adequate to model service interactions:

1) parallelism and communication mechanism in CAPNs, which enable distributed service interaction on internet;

2) CAPNs are compositional, which corresponds to the possibility to build bigger behaviors from small ones;

3) CAPN can be executed, which ensures a executable service interaction environment for actual application;

4) context-awareness semantics in CAPNs accords to context-awareness semantics of web services.

So, we use CAPN to model interaction of contextaware web services.

Figure [I shows the interaction model of contextaware web services, i.e. context-aware web service network. A web service is modeled as a computing process of CAPN. Web services communicate with each other using FIFO channels. The main modeling elements of Context-aware Web Service Network are 


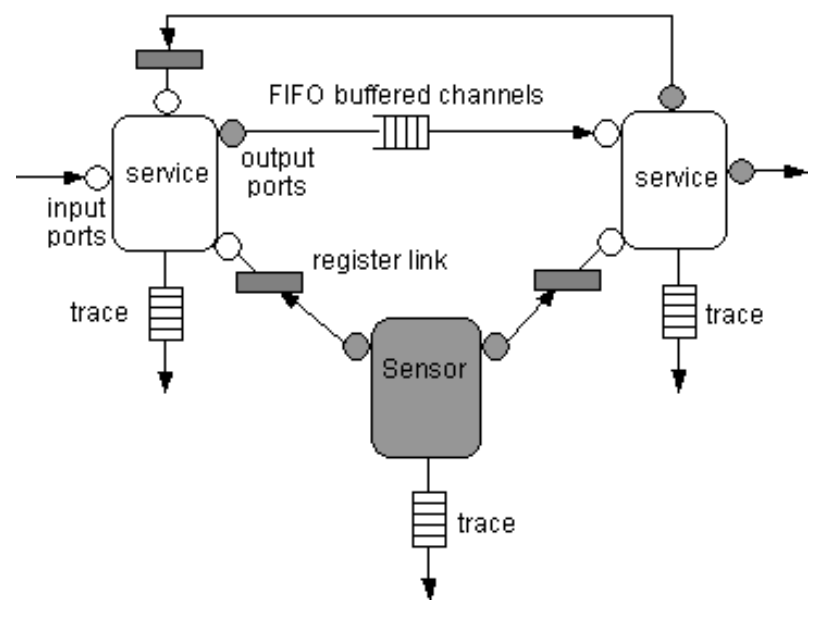

Fig. 1. Context-aware Web Service Network.

service, sensor, input port (denoted as small white circle), output port (denoted as small grey circle), FIFO buffered channels, register link and trace. In this model, in order to simplify the process of context capturing, we add a sensor element which is a kind of additional CAPN processes and only used to get contexts from environment and send them to web services. The normal CAPN process, i.e. computing process is used to deal with application data among web services.

\subsubsection{Modeling Web Service and Sensor Service}

In this model, we model a web service as a computing process of CAPN. A web service is described as a service interface which has a set of service operations. A service operation consists of a number of input and output ports through which the input and output parameters of service operations are passed. We model a sensor service as an additional process. For convenience, a sensor service is defined as a kind of specific web services, similar to a web service in its interface, operation, input and output ports, but different from a web service in its behavior. A sensor service can only link to register links and can't link to FIFO channels, while a web service hasn't this limitation.

\subsubsection{Modeling Behaviors of FIFO Buffered Channels}

A web service communicates with others using FIFO buffered channels which are unbounded queues of data tokens between two services. For each channel there is a single service that produces messages and a single service that consumes messages. Multiple producers or multiple consumers connected to the same channel are not allowed. Data is consumed from these queues in a first-in first-out order. Each service port is linked to at most one other port through a unidirectional unbounded FIFO queue. Services are allowed to read from an input port and are allowed to

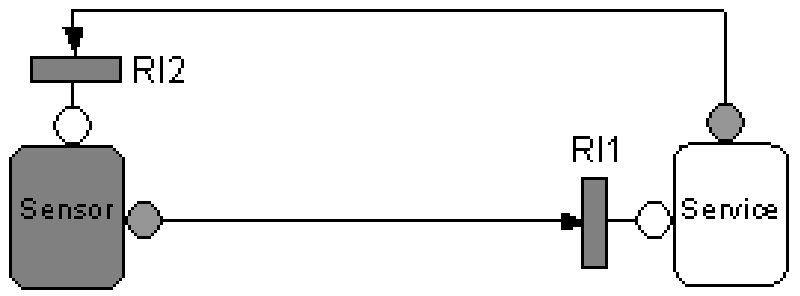

Fig. 2. Register link between a web service and a sensor service.

write to an output port. The read operation is blocking and the write operation is non-blocking. When tokens are available on every input, the service will execute.

\subsubsection{Modeling Behaviors of Register Links}

In this model, register link is also an unidirectional non-blocking channel. But the behavior semantic of a register link and a FIFO buffered channel is very different. In [21], the behavior of a register link is defined as follows.

- Writing to a full register link overwrites a previously written value;

- Reading from a register link returns the last received value;

- A value can be returned multiple times.

In this paper, we only care about the context's influence on web service's behaviors. Register link is used to carry context information between two web services or between a web service and a sensor service. We don't consider the circumstance that register link is between two sensor services.

The circumstance of register link between a web service and a sensor service is depicted in Figure D.

In this circumstance, context information, which is captured by sensor, including network bandwidth, user preference and so on, will be sent to a web service for use. When the web service is to execute, the event of reading from its input register link Rl1 will be triggered, to make sure that the latest contexts are read and used by the web service. After execution, the web service may write other context information to its output register link $R l 2$.

As to the circumstance of register link between two web services, we consider the following case, depicted in Figure B.

In this case, $S 1$ and $S 2$ are two interacting web services. S1 interacts with $S 2$ through FIFO buffered channel $C 1$ and a register link $R l 1$. The execution of $S 1$ will trigger a writing event to $C 1$. When a reading event from $C 1$ finishes, $S 2$ will execute. Also, the execution of $S 2$ will trigger a writing event to $R l 1$, to notify $S 1$ whether the interaction is successful or not. So, the context information in $R l 1$ denotes feedback information from $S 2$. From this feedback information, 


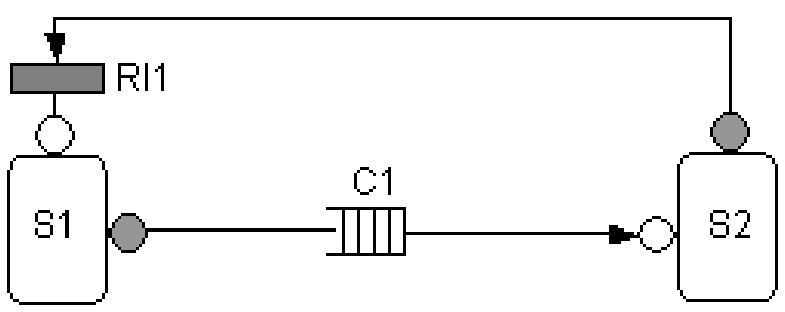

Fig. 3. Register link between two web services.

$S 1$ will decide whether to adjust its control strategy and reply or not.

\subsubsection{Service Interaction Events}

In this model, there are five service interaction events, which are reading from a FIFO buffered channel, writing to a FIFO buffered channel, reading from a register link, writing to a register link and executing a web service. We named these five events as readchannel(), writechannel(), readreg(), writereg() and execute(). For an interacting web service, its behavior trace is composed of four reading/writing events (execute() is a internal event), the event order lies in the computation logic. A single trace denotes the execution of a single web service and a composite trace of all services represents the unrolled execution of contextaware web service network.

\section{Behavior Semantics of Context- aware Web Service Network}

In this Section, based on the works in [40], we describe behavior semantics of context-aware web service network using CCS process algebra [4T], [42]. We add context-awareness, behavior compatibility and some other properties to basic semantics of KPN.

Let $C$ be the set of FIFO channels and for each channel $c \in C$, with a channel alphabet $\Sigma_{c}$. Let $R$ be the set of register links, and for every register link $r \in R$, with a alphabet $\Lambda_{r}$.

Definition 1: [Context-aware Web Service]

A context-aware web service is a labelled transition system

$L T S=\left(S ; s_{0} ; I_{C} ; O_{C} ; I_{R} ; O_{R} ; O p ;\right.$ Pt;Ctxp $;$ Act $\left.\rightarrow\right)$, where

- $S$ is the set of service states;

- $s_{0} \in S$ is the initial service state;

- $I_{C} \subseteq C$ is the set of input channels;

- $O_{C} \subseteq C$ is the set of output channels;

- $I_{R} \subseteq R$ is the set of input register links;

- $O_{R} \subseteq R$ is the set of output register links;

- $O p$ is the set of service operations;

- $P t$ is the set of service ports provided by service operations;

- Ctxp is the context processor which is a software module used to process contexts inside the web service;
- Act represents the set of actions, Act = $\left\{c ? a, c ! a \mid c \in I_{C} \cup O_{C}, a \in \Sigma_{c}\right\} \cup\{r ? b, r ! b \mid r \in$ $\left.I_{R} \cup O_{R}, b \in \Lambda_{r}\right\} \cup\{\tau\}$, where $c$ ? $a$ represents a read action from channel $c$ and $c ! a$ represents a write action to channel $c$ with token $a, r ? b$ represents a read action from register link $r$ and $r ! b$ represents a write action to register link $r$ with token $b,\{\tau\}$ is the internal or silent action (all other actions); $\rightarrow \subseteq S \times A c t \times S$ is a labelled transition system, we write $s_{1} \stackrel{\alpha}{\longrightarrow} s_{2}$ if $\left(S_{1} ; \alpha ; s_{2}\right) \in \rightarrow$.

Definition 2: [Context-aware Web Service Network] A context-aware web service network is a tuple $\left(P ; N_{C} ; N_{R} ; I_{C} ; O_{C} ; I_{R} ; O_{R} ;\right.$ Act $\left.;\{L T S p \mid p \in P\}\right)$, where

- $P$ is the set of web services;

- $N_{C} \in C$ is the set of internal channels (between two services);

- $N_{R} \in R$ is the set of internal register links (between two services);

- $I_{C} \in C$ is the set of input channels;

- $O_{C} \in C$ is the set of output channels;

- $I_{R} \in R$ is the set of input register links;

- $O_{R} \in R$ is the set of output register links;

- Act represents the set of actions, $A c t=\left\{c ? a, c ! a \mid c \in I_{C} \cup O_{C} \cup N_{C}, a \in \Sigma_{c}\right\} \cup$ $\left\{r ? b, r ! b \mid r \in I_{R} \cup O_{R} \cup N_{R}, b \in \Lambda_{r}\right\} \cup\{\tau\}$, where $c ? a, c ! a, r ? b, r ! b,\{\tau\}$ have the same meaning as that in Definition II.

- $L T S_{p}=\left(S_{p} ; s_{p_{0}} ; I_{C_{p}} ; O_{C_{p}} ; I_{R_{p}} ; O_{R_{p}} ; O p_{p} ; P t_{p} ; C t x p_{p}\right.$; Act $p ; \rightarrow)$ denotes the labelled transition sysstem of $p \in P$.

Definition 3: [Configuration] A configuration of a context-aware web service network is a 3-tuple $(\psi, \eta, \zeta)$, where:

- $\psi: P \rightarrow S=\cup_{p \in P} S_{p}$ is a service state function that maps every service $p \in P$ to a local state $s \in S_{p}$, here $S_{p}$ is the state set of service $p$;

- $\eta: C \rightarrow \Sigma^{*}$ is a channel state function that maps every channel $c \in C$ to a finite string $\eta(c)$ on $\Sigma_{c}$, here $\Sigma_{c}$ is the corresponding channel alphabet of $c, \Sigma$ denotes the union of all channel alphabet, $\Sigma^{*}$ denotes the set of all finite (and infinite) strings over $\Sigma$;

- $\zeta: R \rightarrow \Lambda^{*}$ is a register link state function that maps every register link $r \in R$ to a finite string $\psi(r)$ on $\Lambda_{r}$, here $\Lambda_{r}$ is the corresponding register link alphabet of $r, \Lambda$ denotes the union of all register link alphabet, $\Lambda^{*}$ denotes the set of all finite (and infinite) strings over $\Lambda$.

In a context-aware web service network, there exists an initial configuration $\left(\psi_{0}, \eta_{0}, \zeta_{0}\right)$, here $\psi_{0}$ maps every service $p \in P$ to its initial state $s_{p_{0}}, \eta_{0}$ maps every channel $c \in C$ to an empty string and $\zeta_{0}$ maps every register link $r \in R$ to an empty string.

Definition 4: [Behavior Semantics of Channel and Register Link] The reading and writing semantics of FIFO channel and register link are defined as follows: 
1) A service reads from a channel:

$$
\frac{\psi(p) \stackrel{c ? a}{\longrightarrow} s, \eta(c)=a \sigma, c \in C}{(\psi, \eta) \stackrel{c ? a}{\longrightarrow}(\psi[s / p], \eta[\sigma / c])} ;
$$

2) A service writes to a channel:

$$
\frac{\psi(p) \stackrel{c ! a}{\longrightarrow} s, \eta(c)=\sigma, c \in C}{(\psi, \eta) \stackrel{c ! a}{\longrightarrow}(\psi[s / p], \eta[a \sigma / c])},
$$

Here $\sigma \in \Sigma^{*}$ and $\psi[s / p]$ is the function with the same domain as $\psi$ except that $\psi(p)=s . \eta[\sigma / c])$ is the function with the same domain as $\eta$ except that $\eta(c)=\sigma$;

3) A service reads from a register link:

$$
\frac{\psi(p) \stackrel{r ? b}{\longrightarrow} s, \zeta(r)=b \rho, r \in R}{(\psi, \zeta) \stackrel{r ? R}{\longrightarrow}(\psi[s / p], \zeta[b \rho / r])},
$$

Here, we assume $b$ is the last received value in register link $r$. If $b$ hasn't been rewritten by a writing event, it is still in register link for further reading. And, $\rho \in \Lambda^{*}, \psi[s / p]$ is the function with the same domain as $\psi$ except that $\psi(p)=s$, $\zeta[\rho / r]$ is the function with the same domain as $\zeta$ except that $\zeta(r)=\rho$;

4) A service writes to a register link:

$$
\frac{\psi(p) \stackrel{r ! b}{\longrightarrow} s, \zeta(r)=\rho, r \in R}{(\psi, \zeta) \stackrel{r ! R}{\longrightarrow}(\psi[s / p], \zeta[b \rho / r])},
$$

Here, we assume register link $r$ isn't full. When $r$ is full, let $b_{0}$ is the last token in register link $r$, the above expression will be:

$$
\frac{\psi(p) \stackrel{r ! b}{\longrightarrow} s, \zeta(r)=\rho b_{0}, r \in R}{(\psi, \zeta) \stackrel{r ! R}{\longrightarrow}(\psi[s / p], \zeta[b \rho / r])},
$$

That is, after $b$ is written to the full register link $r$, the last token $b_{0}$ in register link $r$ has been rewritten.

Definition 5: [Execution of Context-aware Web Service Network] A labelled transition system $L T S$ is defined as a tuple $\left(Z ; z_{0} ; N_{C} ; N_{R} ; I_{C} ; O_{C} ; I_{R} ; O_{R} ; A c t ; \rightarrow\right.$ ), where:

- $Z$ is the set of all configurations;

- $z_{0} \in Z$ is the initial configuration;

- $N_{C} \subseteq C$ is the set of internal channels (between two services);

- $N_{R} \subseteq R$ is the set of internal register links (between two services);

- $I_{C} \subseteq C$ is the set of input channels;

- $O_{C} \subseteq C$ is the set of output channels;

- $I_{R} \subseteq R$ is the set of input register links;

- $O_{R} \subseteq R$ is the set of output register links;

- Act represents the set of actions, the meaning is the same as that in Definition 2 ;

- $\rightarrow \subseteq Z \times$ Act $\times Z$ is a labelled transition relation, we write $z_{1} \stackrel{\alpha}{\longrightarrow} z_{2}$ if $\left(z_{1} ; \alpha ; z_{2}\right) \in \longrightarrow$.
An execution of a context-aware web service network is a sequence $k=z_{0} \stackrel{\alpha_{0}}{\longrightarrow} z_{1} \stackrel{\alpha_{1}}{\longrightarrow} z_{2} \stackrel{\alpha_{2}}{\longrightarrow}$ $\cdots z_{i} \stackrel{\alpha_{i}}{\longrightarrow} \cdots$ of configurations, $z_{i} \in Z$ and action $\alpha_{i}$ Act, such that $z_{i} \stackrel{\alpha_{i}}{\longrightarrow} z_{i+1}$ for all $i \geq 0$. Every execution step $z_{i} \stackrel{\alpha_{i}}{\longrightarrow} z_{i+1}$ denotes a synchronization point of interacting web services in a context-aware web service network.

Definition 6: [Behavior Trace and Composite Behavior Trace] In an execution of a context-aware web service network, as described in Definition $\square$, we define a behavior trace of an interacting context-aware web service $p$ as a finite action sequence $\lambda_{p}=$ $\alpha_{p 1} \alpha_{p 2} \cdots \alpha_{p i} \cdots \alpha_{p n}$, where $\alpha_{p i} \in$ Act, Act represents the set of reading or writing actions, the meaning is the same as that in Definition ㄴ, $1 \leq i \leq n$, $n \in N, N$ is the set of positive integers. We define the finite sequence $\lambda=\alpha_{0} \alpha_{1} \cdots \alpha_{i} \cdots$ in execution $k=z_{0} \stackrel{\alpha_{0}}{\longrightarrow} z_{1} \stackrel{\alpha_{1}}{\longrightarrow} z_{2} \stackrel{\alpha_{2}}{\longrightarrow} \cdots z_{i} \stackrel{\alpha_{i}}{\longrightarrow} \cdots$ as a composite behavior trace of this network. Composite behavior trace represents the unrolled execution of this network. We use $\operatorname{trace}(p)$ to denote behavior trace of an interacting context-aware web service $p$.

Behavior traces can be used to analyse and verify the behaviors of interacting web services. Behavior traces as behavior description of a web servcie can serve as inputs to related verification tools (e.g., CWBNC [42]), where the service behavior represented in the description can be analysed and verified.

In a context-aware web service network, we consider contexts in register links as a kind of controlling information to adjust internal control flow of a web service, not as the parameters of a web service. Only when a web service is to execute, it reads contexts from register links. So if we don't consider the influence of contexts, this network becomes corresponding KPN. Next we define behavior compatibility of two interacting web services, we only consider writing/reading actions on internal FIFO channels between this two interacting web services.

Let $Q$ is the set of web services, $q_{0} \in Q, q_{i} \in Q$, where $1 \leq i \leq n, n \in N, N$ is set of positive integers.

Definition 7: [Weak Transitions] Let $q \in Q, q^{\prime} \in Q$, $\tau$ is transition label for action which is not externally visible, if

- $q \Rightarrow^{\varepsilon} q^{\prime}$ iff $q=q_{0}=\rightarrow^{\tau} q_{1} \rightarrow^{\tau} \cdots \rightarrow^{\tau} q_{i} \rightarrow^{\tau}$ $\cdots \rightarrow^{\tau} q_{n}=q^{\prime}, n \geq 0$

- $q \Rightarrow^{\tau} q^{\prime}$ iff $q \Rightarrow^{\varepsilon} q^{\prime}$;

- $q \Rightarrow^{\alpha} q^{\prime}$ iff $q \Rightarrow^{\varepsilon} q_{1} \rightarrow^{\alpha} q_{2} \Rightarrow^{\varepsilon} q^{\prime}(\alpha \neq \tau)$

then, $q \Rightarrow^{\alpha} q^{\prime}$ is a weak transition.

Definition 8: [Observational Equivalence] Let $S \subseteq$ $Q \times Q$, the relation $S$ is a weak bisimulation relation if whenever $q_{1} S q_{2}$ then:

- if $q_{1} \rightarrow^{\alpha} q_{1}^{\prime}$, for some $q_{2}^{\prime}$, implies $q_{2} \Rightarrow^{\alpha} q_{2}^{\prime}$, and $q_{1}^{\prime} S q_{2}^{\prime}$

- if $q_{2} \Rightarrow^{\alpha} q_{2}^{\prime}$, for some $q_{1}^{\prime}$ implies $q_{1} \Rightarrow^{\alpha} q_{1}^{\prime}$, and $q_{1}^{\prime} S q_{2}^{\prime}$; then, $q_{1}$ and $q_{2}$ is observationally equivalent, or weakly bisimulation equivalent, 
that is, for an external observer, it is not possible to distinguish the behavior of $q_{1}$ and $q_{2}$.

Definition 9: [Behavior Compatibility] Two interacting web services $p$ and $q$ are behavior compatible, denoted as compatible $(p, q)$, if they have opposite behavior trace (only including reading/writing actions on FIFO channels between this two web services), i.e., $p$ is Observationally Equivalent to $q$.

For example, let '!' denotes writing action, writing $P(p)$ denotes the writing token sequence of service $p$, reading $Q(q)$ denotes the reading token sequence of service $q$. Let $\operatorname{trace}(p)=$ !Req.Receive.nil, $\operatorname{trace}(q)=$ Req.!Receive.nil, it's clear that, writing $P(p)=$ reading $Q(q)$ and $w$ riting $Q(q)=$ reading $P(p)$ when $p$ and $q$ execute a reading/writing action respectively, that is $p \stackrel{! R e q}{\longrightarrow} p^{\prime}, q \stackrel{\operatorname{Req}}{\longrightarrow} q^{\prime}$, then, $p^{\prime}=$ Receive.nil, $q^{\prime}=$ !Receive.nil. It's clear writing $P\left(p^{\prime}\right)=$ reading $Q\left(q^{\prime}\right)$ and writing $Q\left(q^{\prime}\right)=$ reading $P\left(p^{\prime}\right)$ when $p^{\prime}$ and $q^{\prime}$ execute a reading/writing action respectively, that is $p^{\prime} \stackrel{\text { Receive }}{\longrightarrow}$ nil, $q^{\prime} \stackrel{! \text { Receive }}{\longrightarrow}$ nil, $p^{\prime}$ and $q^{\prime}$ will be nil. It's clear that, behaviors of $p$ and $q$ is opposite. So they are behavior compatible.

When a context-aware web service network is executing, deadlock may be occurred. In [43], Parks distinguishes between two types of deadlocks. 'True' deadlocks are those described in Kahn's model, that is all processes are blocked on reads and all channels are empty, then the computation has terminated. 'Artificial' deadlocks are caused by bounded channel capacities. Since channel capacities are not restricted in Kahn's model, artificial deadlock is impossible.

In a context-aware web service network, a deadlock can only be 'True' deadlock. Since both FIFO Buffered Channels and Register Link have unbounded size.

Theorem 1: [Undecidable] The problem of whether a context-aware web service network will terminate is undecidable.

Proof: Kahn Process Network can be thought of as a set of Turing machines connected by one-way tapes. It is impossible to decide whether or not an arbitrary Turing machine program will halt [43], [44]. So it is undecidable if a Kahn Process Network will terminate. This implies the fact that it is undecidable whether or not a context-aware web service network will terminate.

Let $I=I_{C} \cup I_{R}, O=O_{C} \cup O_{R}$, in an execution $k$ of this network, we define an input function in $: I \rightarrow \Sigma^{*} \cup \Lambda^{*}$ that maps every input channel to a finite (and infinite) strings on $\Sigma^{*}$ and every input register link to a finite (and infinite) strings on $\Lambda^{*}$. We define a function out : $O \rightarrow \Sigma^{*} \cup \Lambda^{*}$ that maps every output channel to a finite (and infinite) strings on $\Sigma^{*}$ and every output register link to a finite (and infinite) strings on $\Lambda^{*}$. For some $c \in I$, we use $k$ ? $c$ to denote the input consumed on $c$ and $k$ ? I to denote the input consumed by the network in execution $k$. For some $o \in O$, we use $k ! o$ to denote the output on $o$ and $k ! O$ denotes the output of the network in execution $k$.

Definition 10: [Completeness] An execution $k$ of a context-aware web service network with an input in: $I \rightarrow \Sigma^{*} \cup \Lambda^{*}$ is complete, denoted as complete $(k)$, iff, the execution of corresponding KPN is complete [43], i.e.

- $\forall p \in P, P$ is the set of web services, $\exists n \in N$, $N$ is the set of positive integers, such that $0 \leq$ $|\operatorname{trace}(p)| \leq n$.

- $\forall o \in O,(k ! o) / R=\operatorname{out}(o) / R,(k ! o) / R$ denotes the output of the context-aware web service network in execution (taking out those outputs produced by actions involving register links set).

That is in the execution $k$ of corresponding KPN, every behavior trace of web service has a finite number of actions, and none of the output can be extended again.

Definition 11: [Effectiveness] Here, we add the support of register links to the definition of Maximality and Effectiveness of an execution of KPN in [40] and define Effectiveness of an execution of a context-aware web service network. An execution $k$ of a contextaware web service network with an input in $: I \rightarrow$ $\Sigma^{*} \cup \Lambda^{*}$ is effective, denoted as $\operatorname{effective}(k)$, iff,

- at the last configuration, only read actions are possible, and all inputs of input in $: I \rightarrow \Sigma^{*} \cup \Lambda^{*}$ have been consumed entirely (Maximality in [40], here, we consider not only FIFO channels but also register links), i.e. if $n \in N$ is the length of the execution $z_{n} \stackrel{\alpha}{\rightarrow}$, then $\alpha=c$ ? $a$ and $k ? c=i n(c)$ for some $c \in I_{C} \cup I_{R}$ and $a \in \Sigma_{c} \cup \Lambda_{r}$.

- every token produced in internal channels and internal register links is ultimately consumed (Effectiveness in [40], here, we consider not only FIFO channels but also register links), i.e. $k ? N_{C}=$ $k ! N_{C}$ and $k ? N_{R}=k ! N_{R}$, where $N_{C}$ is the set of internal channels and $N_{R}$ is the set of internal register links.

Theorem 2: [Comp ${ }^{2}$ ] In an execution $k$ of a contextaware web service network, if effective $(k)$, then complete $(k)$ and compatible $(p, q)$, for some $p, q \in P$, $P$ is the set of web services in this network. Proof:

1) We first prove complete $(k)$ can satisfy. An execution $k$ of a context-aware web service network is effective states that two conditions in Definition 10 are satisfied. The first condition of Definition 10 implies that at the last configuration, execution $k$ finishes and only a new execution with a new input can execute, that is, $\forall p \in P$, we assume $k=z_{0} \stackrel{\alpha_{0}}{\longrightarrow} z_{1} \stackrel{\alpha_{1}}{\longrightarrow}$ $\cdots z_{i} \cdots \stackrel{\alpha_{i}}{\longrightarrow} z_{j}$, then $\operatorname{trace}(p)=\alpha_{1} \alpha_{2} \cdots \alpha_{j}$, there must $\exists n \in N, j \leq n,|\operatorname{trace}(p)|=j$. This satisfies the first condition of completeness definition in Definition [10. Also, at the last configuration, the fact that execution $k$ finishes implies that none 
of the output can be extended again, that is $\forall o \in O,(k ! o) / R=\operatorname{out}(o) / R$. This satisfies the second condition of completeness definition in Definition [10.

2) We prove compatible $(p, q)$ can satisfy. $\forall p, q \in P$, $p$ and $q$ is two interacting web services, let $\operatorname{trace}(p)=\alpha_{1} \alpha_{2} \cdots \alpha_{j}$, $\operatorname{trace}(q)=\beta_{1} \beta_{2} \cdots \beta_{j}$. According to behavior compatibility in Definition 9 , we only need to consider writing/reading actions on internal channels between this two services. For an internal channel, writing action precedes its opposite reading action. The second condition of Definition 101 implies that, if $\alpha_{1}=c ! a$ is a writing action in $\operatorname{trace}(p)$, there must exist $\beta_{i}$ in $\operatorname{trace}(q)$ is the opposite reading action of $\alpha_{1}$. Because a channel is a FIFO queue, the token $a$ in channel $c$ must be read first in $\operatorname{trace}(q)$. So $\beta_{i}$ must be the first action in $\operatorname{trace}(q)$, that is $\beta_{i}=\beta_{1}$. For the same reason, $\beta_{2}$ is the opposite action of $\alpha_{2}, \beta_{i}$ is the opposite action of $\alpha_{i}$, and so on, finally $p$ and $q$ produce the opposite behavior trace. This satisfies the behavior compatibility in Definition Q9, that is compatible $(p, q)$ is satisfied.

\section{Behavior Adaptation of Web Ser- VICES}

Context-awareness allows a web service to adapt to changes of their contexts. In order to realize this goal, we define a context processor for every context-aware web service. A context processor is a software module used to collect and process every context input to a context-aware web service. According to the basic semantic of a context-aware web service network, when tokens are available on every input, the web service will execute. Now, we consider the influence of contexts on this web service. When a web service is to be executed, the execute() event will trigger its context processor to work in advance, as depicted in Figure 田.

The context processor reads from each register link in terms of its reading semantic, and puts them into a context file. Then, the context processor is responsible for managing and updating this file. A web service may contain lots of context types and their values. Also different web service contains different contexts. So we model context information as a XML formatted file which provides a unified description format for different contexts. The platform independent feature of XML can easily tackle the heterogeneity of different contexts. A web service uses such context information to adjust its internal control flow as well as content and format of its replies.

The context file of a web service is composed of elements of context type. A context type contains a context value and a context weight. The basic format

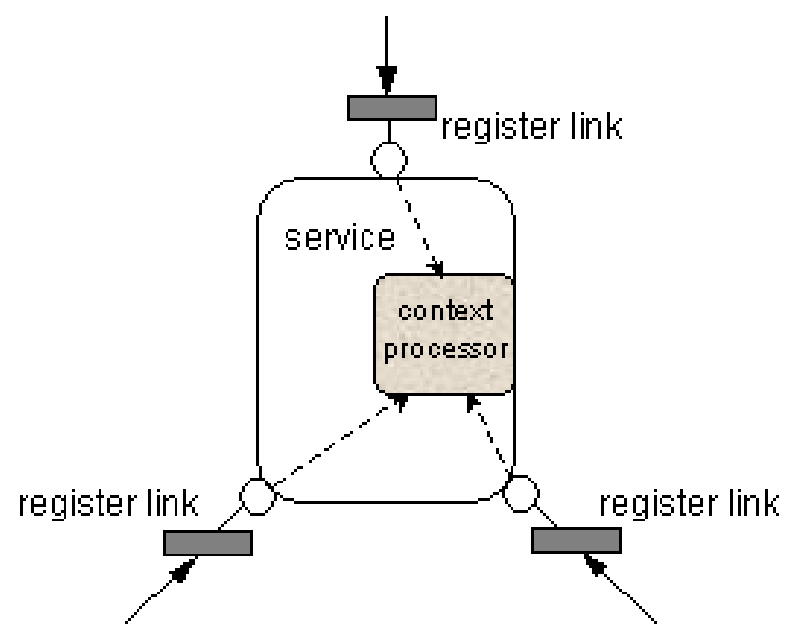

Fig. 4. Context processor of a web service.

TABLE 1

A context file of a web service.

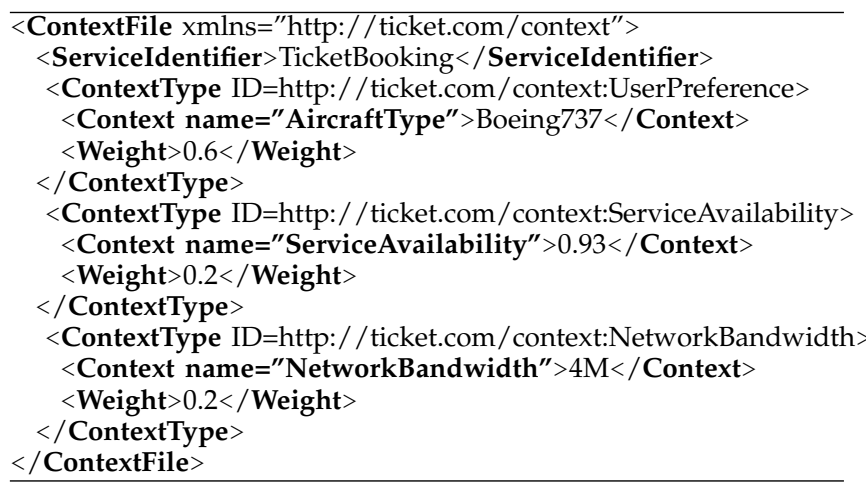

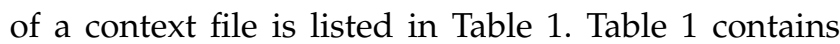
three context types which are User Preference, Service Availability and Network Bandwidth.

During the execution of a web service, it responds to each context in context file. The basic context processing process is as follows.

1) Parse context file using XML parser,

2) For each context in context file,

3) Calculate weight or influence on this web service,

4) Adjust internal control flow and reply of this web service according to all contexts.

We use three kinds of contexts to characterize the situation of service interactions, including interaction environment context (network bandwidth, operating system, client's devices, etc), user context (user preference, user location, etc) and service context (business restriction, service availability, execution time, success rate, etc). In actual application, we can obtain these context values with various approaches. For example, network bandwidth cab be got through related 
network bandwidth monitoring software, service execution time can be obtained through web log, user preference can be expressed by user when interacting with web service and service availability can be achieved by calculating the average available time of a web service in the past period of time, etc.

Then how to calculate the influence of contexts on a web service? we know that a context-aware web service has to accommodate for a variety of context types. So we need to calculate influences on a web service in different manner according to the characteristics of each context type. For example, network bandwidth may influence the execution of a web service, in case of a narrow bandwidth the web service may terminate, in case of a normal bandwidth the web service can output the expected result. User preference context may influence the output of web service, and we can use this context to filter the output of the web service, thus getting a new output meeting the user preference. In addition, the value type of each context is also different, some is integer, some is string, etc, so we need to convert them to a uniform format for convenience of calculations.

We define an influence function $f(C)$ for every context type, denoting the influence of context $C$ on a web service, whose value type is numeric and ranges from 0 and 100 or others according to the actual application. We also assign a weight $w$ whose value is ranging from 0 and 1 for each context $C$, denoting the influence degree of this context type.

As discussed above, for a context-aware web service, behavior adaptation is its capability to provide different versions of behaviors to meet the needs of context changes. We define different behavior versions for a context-aware web service according to the influences of different context types and their values. In this paper, we assume the influence function and weight is the same in different behavior versions for a certain context type. So we only need to differentiate context types and their values in different behavior versions. We use a XML formatted file to describe different behavior versions of a context-aware web service. The following is an example.

$<$ behaviorVersion1 $>$

$<$ UserPreference AircraftType="Boeing737" / >

$<$ ServiceAvailability $>0.9</$ ServiceAvailability $>$

$<$ NetworkBandwidth $>4 \mathrm{M}</$ NetworkBandwidth $>$

$</$ behaviorVersion1 $>$

$<$ behaviorVersion2 $>$

$<$ UserPreference AircraftType="Boeing747" / >

$<$ ServiceAvailability $>0.8</$ ServiceAvailability $>$

$<$ NetworkBandwidth $>2 \mathrm{M}<$ /NetworkBandwidth $>$ $</$ behaviorVersion2 $>$

Assuming a web service has $n$ behavior versions denoted as $s_{1}, s_{2}, \ldots \ldots, s_{n}$, then we use the following formula to calculate the predefined rank for each version (one version with a unique predefined rank).

$$
\operatorname{Rank}=\sum_{i=1}^{n} f_{i}\left(C_{i}\right) * w_{i}
$$

where $f_{i}\left(C_{i}\right)$ is the influence function of context $C_{i}$, $w_{i}$ is the weight of context $C_{i}, 1 \leq i \leq n, n$ is a positive integer. When the current context values of a web service are obtained, we can calculate the rank of current context situation using the formula above. From the predefined behavior versions, we can select an appropriate one whose predefined rank is equal or closest to the calculated one.

In an actual application, we can adjust internal control of a web service by adjusting its behavior versions. If there exists a large discrepancy between the rank of current context situation and all predefined ranks, we need to adjust the behavior versions of this web service statically or dynamically to make sure an appropriate behavior version can be selected. For example, we may add a new behavior version when one or more new context types are added. We may remove a behavior version when related context types are not useful. We may also modify a behavior version by modifying related context types and their values or modifying related context influence functions and their weights. We may define a context influence function and specify its weight for a context with experiential values at the very beginning, and then adjust them dynamically and iteratively according to the reply of web service.

\section{A XML Formatted Service Behavior DESCRIPTION LANGUAGE}

Based on the basic semantics of context-aware web service network, we design a XML formatted service behavior description language named BML4WS (Behavior Modeling Language for Web Service) to describe behaviors and behavior adaptation of interacting context-aware web services. Because contexts in register links aren't the parameters of a web service only as a kind of controlling information to adjust internal control flow of a web service, if we don't consider the influence of contexts, this network becomes corresponding KPN. According to KPN semantics, we can execute the KPN network in parallel, as well as sequentially. So we consider web services in contextaware web service network as sequential processes in BML4WS.

\subsection{Structure of BML4WS}

The basic structure of this description language is as follows:

$<$ bml:process $>$

$<$ bml:service name $=$ "service name" $>$

$<$ !-describing service operations $->$

$<$ bml:operation name $=$ "operation name" $>$

$<$ !-describing input ports of service operation $->$ $<$ bml:input $>$

name" /> $<$ bml:port name $=$ "port name" type="type

$</$ bml:input $>$

$<$ !-describing output ports of service operation ->

$<$ bml:output $>$ $<$ bml:port name="port name" type="type 
name" $/>$

$</$ bml:output $>$

......

$</$ bml:operation $>$

$</$ bml:service $>$

$<$ bml:service name $=$ "service name" $>\cdots+</$ bml:service $>$

......

$<!-$ defining channels $->$

$<$ bml:channel $>$ channel name $</$ bml:channel $>$

.......

$<!-$ defining register links $->$

$<$ bml:reg $>$ register link name $</$ bml:reg $>$

$<$ !-describing behavior interaction $->$

$<$ bml:composition $>$

$<!-$ describing sequence interactions $->$

$<$ bml:sequence $>$

$<$ !-reading from a channel $->$

$<$ bml:readchannel channel="channel name"

service $=$ "service name" operation $=$ "operation name"

port="port name" />

.......

$<$ !-executing a service operation $->$

$<!-A t$ this moment, context processor of this web service will read from each register link and form context file, then context file will be processed by this web service $->$

$<$ bml:readreg reg="register name" service $=$ "service name" operation="operation name" port="port name" />

$<$ bml:execute operation="operation name"

service $=$ "service name" $/>$

$<$ !-writing to a channel $->$

$<$ bml:writechannel channel="channel name" service $=$ "service name" operation $=$ "operation name" port="port name" />

......

$<!-$ writing to a register link $\rightarrow>$

$<$ bml:writereg reg="register name" service $=$ "service name" operation="operation name" port="port name"/>

$</$ bml:sequence $>$

…...

$<$ bml:loop $>\cdots+</$ bml:loop $>$

$</$ bml:composition $>$

$<$ bml:process $>$

\subsection{BML4WS Interpreter}

In order to evaluate and validate the service behavior modelling approach presented in this paper. We design a BML4WS interpreter to implement behavior interactions among web services. The architecture of BML4WS interpreter is described in Fig. ‥ BML4WS interpreter is composed of three main components, which are BML4WS Document Parser, Scheduler for Service Interaction Events and Service Interaction APIs.

- BML4WS Document Parser is used to load and parser a BML4WS service behavior description file, the DOM (Document Object Model) tree of BML4WS file is depicted in Fig. 6. The output is composite behavior trace of the whole system represented by this description file. Algorithm
W shows the generation algorithm of composite behavior trace.

- Scheduler for Service Interaction Events is used to schedule and execute every service interaction event in composite behavior trace. Algorithm [ shows the Scheduling algorithm of the composite behavior trace.

- Service Interaction APIs is the Java implementation of five service interaction events which are readchannel(), writechannel(), readreg(), writereg() and execute().

Algorithm 1 BuildCompositeTrace(BML4WSFile)

Inputs: BML4WSFile: a BML4WS service behavior description file

Outputs: actionList: a composite behavior trace of the system represented by BML4WSFile

01 ArrayList $<$ ActionType $>$ actionList $=$

01 new ArrayList();

02 ActionType action=null;

03 for all element in BML4WSFile do

04 if(element.getName.equals("composition")) then

05 for all subelement of element do

06 action= buildAction(subelement);

06 / / add into actionList

07 actionList.add(action);

08 end for

09 endif

10 end for

11 return actionList

BuildAction(element)

Inputs: an element of BML4WS Document

Outputs: action type: one of readchannel, writechannel, readreg, writereg and execute

12 elname=element.getName;

13 if (elname.equals("readchannel")) then

14 ReadChannel readChannel =

14 new ReadChannel();

15 return readChannel;

16 elseif(elname.equals("writechannel")) then

17 WriteChannel writeChannel =

17 new WriteChannel();

18 return writeChannel;

19 elseif(elname.equals("readreg")) then

20 ReadReg readReg = new $\operatorname{ReadReg}() ;$

21 return readReg;

22 elseif(elname.equals("writereg")) then

23 WriteReg writeReg = new WriteReg();

24 return writeReg;

25 elseif(elname.equals("execute")) then

26 Execute execute = new Execute();

27 return execute;

28 endif

We implement five service interaction events using Java RMI (Remote Method Invocation) and Java stream programming technologies. 

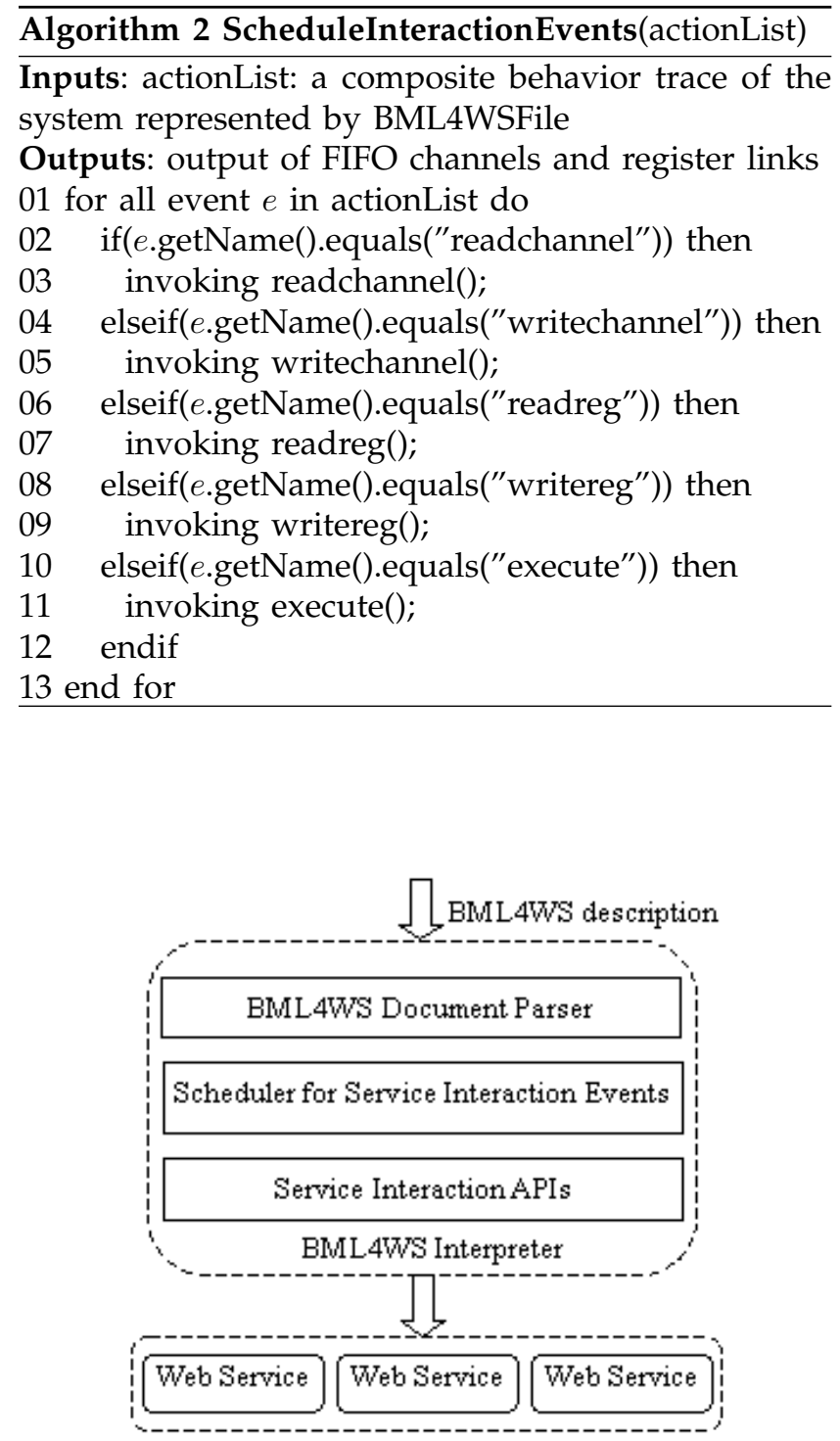

Fig. 5. Architecture of BML4WS interpreter.

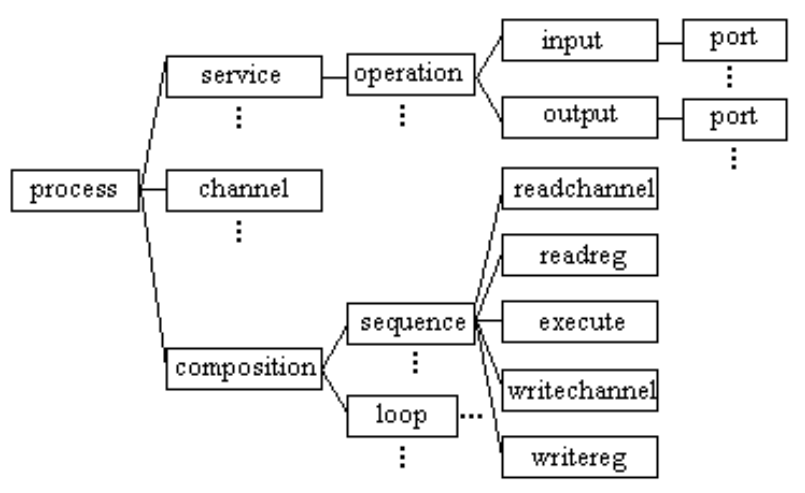

Fig. 6. DOM Tree of BML4WS file.

\section{Case Study}

\subsection{A Context-aware Ticket Booking System}

We design a Context-aware Ticket Booking system, which is composed of four interacting web services, i.e. Client service, Customer service, Order service and Inventory service. We use visual BML4WS Editor implemented by ourselves to design the Contextaware Ticket Booking system, as shown in Fig. $\square$.

The basic business process of this Ticket Booking system is as follows:

1) A Client service collects booking requests from users through channel 'coll' and form a FIFO queue of request messages.

2) The Client service reads from channel 'coll' and sends a request message through channel 're$\mathrm{qu}^{\prime}$ to Customer service for booking tickets. A request message represents a ticket booking request which is a XML formatted file, describing three booking parameters which are departure station, destination station and date.

3) The Customer service receives a request message and sends message through channel 'ask' to Order service to ask creating order.

4) The Order service sends request to Inventory service through channel 'quer' for querying the ticket stock.

5) The Inventory service retrieves and sends the ticket stock result to Order service through channel 'resu'.

6) If the tickets have been sold out, the order will not be created, Order service will send a rejection message to Customer service through channel 'con', and Customer service sends the rejection message to Client service through channel 'repl'.

7) If the tickets is Available, the order and booking result will be created, Order service sends booking result (order information) to Client service through channel 'info'.

8) Return to 2) to deal with another ticket booking request.

9) Return to 1) to deal with another queue of booking request messages.

In this application, if we don't consider the influence of context information on every service, the Order service will send user a XML file which includes all ticket booking results according to the booking parameters.

Now, we consider the influence of contexts on this system. Many context information can be usable in this Ticket Booking system, including User Preference, User Feedback, Service Availability, User Location and Network Bandwidth, etc. Assuming that we only concern about three types of context information, which are User Preference (user context, UP for short), Service Availability (service context, SA for short) and 


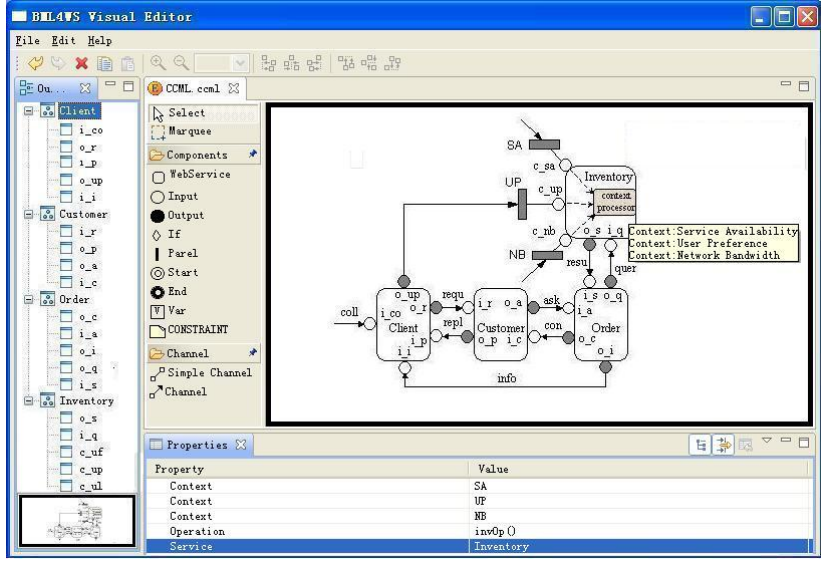

Fig. 7. A Context-aware Ticket Booking System.

Network Bandwidth (interaction environment contex$t$, NB for short). These three types of contexts will be used by Inventory service when ticket stock are retrieved, as depicted in Figure $\square$.

In this case, User Preference context comes from the current user's input, Service Availability context is obtained by calculating the average available time of this web service in the past period of time (second) and Network Bandwidth context comes from related network bandwidth monitoring software. User Preference context will influence output result of the web service, Network Bandwidth and Service Availability context will influence the execution efficiency of the web service.

In order to unify and standardize different value type of contexts, for every context, we define an influence function $f: C \rightarrow N$ that maps a context value $x \in C$ on a score $n$, where, $C$ is a set of context values, $n \in N, N$ is the set of values ranging from 0 to 100 . We define the following context influence function experientially for every context.

For User Preference context, $f_{u p}=100$, for every $c \in C, C$ is the set $\{$ "Boeing737", "Boeing747", ......

For Service Availability context, $f_{s a}=100 * c, 0 \leq$ $c \leq 1$.

For Network Bandwidth context,

$$
f_{n b}=\left\{\begin{array}{l}
100, c>=4 \\
80,1 \leq c<4 \\
60,0<c<1 \\
0, c=0
\end{array} .\right.
$$

We also need to specify a weight for every context. According to the importance degree of every context, we specify the experiential weights as $0.6,0.2,0.2$ for User Preference, Service Availability and Network Bandwidth. In actual situation, we may dynamically adjust these influence functions and related weights according to the reply of web service. Using the rank calculation formula in section 5 , we can calculate the predefined ranks for some frequently used behavior versions, for example, a rank of a main behavior version may be Rank $=100^{*} 0.6+0.2^{*} 90+0.2 * 80=$
94. In current situation, for User Preference context, the current user can decide the actual context value through user interface provided by Client service. For example, the user may select an expected aircraft type, such as Boeing737, by clicking a CheckBox item through user interface provided by Client service. The real-time context value is passed to Inventory service through register link UP. For Network Bandwidth and Service Availability context, they are collected dynamically by context processor of Inventory service from their own register link. Then, context value and its weight are stored into a context file. Using all context values and their weights, the rank of behavior version under current situation can be calculated. According to the calculated rank, the context processor will select a matched behavior version from the predefined behavior versions whose rank is equal or closest to the calculated one to execute.

\subsection{Describing Service Interaction Using BM- L4WS}

In this section, we use BML4WS to describe behaviors of interacting context-aware web services. The corresponding BML4WS file can be exported from visual BML4WS Editor. Here, we only describe interaction between Order service and Inventory service.

$<$ bml:process $>$

$<$ !- Describing Order service and Inventory service- $->$

$<$ bml:service name $=$ "Order" $>$

$<$ bml:operation name $=$ "ordResuOp" $>$

$<$ bml:input $>$

$<$ bml:port name $=" i_{s}$ " type $=" x m l " \mid>$

$</$ bml:input $>$

$<$ bml:output $>$

$<$ bml:port name $=" o_{c}$ " type $=" x \mathrm{ml} " />$

$<$ bml:port name $=" o_{i}$ " type $=" x \mathrm{ml} " />$

$</$ bml:output $>$

$</$ bml:operation $>$

$</$ bml:service $>$

$<$ bml:service name $="$ Inventory" $>$

$<$ bml:operation name $="$ invOp" $>$

$<$ bml:input $>$

$<$ bml:port name $=" i_{q}$ " type $=" x \mathrm{ml} " />$

$</$ bml:input $>$

$<$ bml:output $>$

$<$ bml:port name $=" o_{s}$ " type $=" x \mathrm{xl} " \mid>$

$</$ bml:output $>$

$</$ bml:operation $>$

$</$ bml:service $>$

$<$ !- defining channels and register links ->

$<$ bml:channel $>$ quer $</$ bml:channel $>$

$<$ bml:channel $>$ resu $</$ bml:channel $>$

$<$ bml:channel $>$ info $</$ bml:channel $>$

$<$ bml:reg $>\mathrm{SA}</$ bml:reg $>$

$<$ bml:reg $>\mathrm{UP}</$ bml:reg $>$

$<$ bml:reg $>\mathrm{NB}</$ bml:reg $>$

$<!-$ Describing interaction between Order service and

Inventory service $\rightarrow>$

$<$ bml:composition $>$

$<$ bml:loop $>$

$<$ bml:sequence $>$

$<$ !-here, we omitted the description of writing to channel quer $\rightarrow>$

$<!-$ reading from channel quer-> 


\author{
$<$ bml:readchannel channel="quer" \\ vice $=$ "Inventory" operation $=$ "invOp" port $="{ }^{\prime \prime} i_{q} " />$ \\ $<!-n e x t$, service operation invOp will execute. At this \\ moment, context processor of this web service will read from \\ each register link and form context file, then context file will be \\ processed by this web service $->$ \\ $<$ bml:readreg reg="SA" service="Inventory" \\ operation $=$ "invOp" port $=" c_{s} a^{\prime \prime} />$ \\ $<$ bml:readreg channel $=$ "UP" service $=$ "Inventory" \\ operation $=$ "invOp" port $=" c_{u} p^{\prime \prime} />$ \\ $<$ bml:readreg channel="NB" service $=$ "Inventory" \\ operation $=$ "invOp" port $=" c_{n} b^{\prime \prime} />$ \\ $<$ bml:execute operation="invOp" \\ vice $=$ "Inventory" $/>$ \\ $<$ bml:writechannel channel="resu" \\ ser- \\ vice $=$ "Inventory" operation $=$ "invOp" port $=" o_{s}{ }^{\prime \prime} />$ \\ $<$ bml:readchannel channel $=$ "resu" service $=$ "Order" \\ operation="ordResuOp" port $=" i_{c}$ " / > \\ $<!-$ executing a service operation ordResuOp $\rightarrow>$ \\ $<$ bml:execute operation="ordResuOp" \\ service $=$ "Order" $/>$ \\ $<$ !-writing to channel info- $>$ \\ $<$ bml:writechannel channel $=$ "info" service $=$ "Order" \\ operation="ordResuOp" port $=" o_{i}$ " / > \\ $</$ bml:sequence $>$ \\ $</$ bml:loop $>$ \\ $</$ bml:composition $>$ \\ $</$ bml:process $>$
}

\subsection{Execution and Analysis of Ticket Booking System}

When BML4WS document of the Ticket Booking system is exported, we can use BML4WS Interpreter to Interpret and execute it. We can also use related definitions and theorems presented in section 4 to analyze and verify the properties of this system. For example, we assume an effective execution of Ticket Booking system with an input in $=\left[x_{1}, x_{2}, x_{3}\right], x_{1}, x_{2}$ and $x_{3}$ denote three XML formatted ticket booking requests.

From the BML4WS description, we can extract composite behavior trace of the system using Algorithm 1 . From composite behavior trace of the system, we can extract single behavior trace for every web service. If we only process a booking request, the behavior traces of Order service and Inventory service are as follows:

trace $($ Order $)=$ ask.!quer.resu.(!con+!info)

trace $($ Inventory $)=$ quer.SA.UP.NB.!resu

From Theorem $\square$ we know that an effective execution implies a complete execution and any two interacting web services in this system are behavior compatible. In this complete execution of the Ticket Booking system, three requests must be consumed entirely and every behavior trace has a finite number of actions, as follows:

trace $($ Order $)=$ ask.!quer.resu.(!con+!info).ask .!quer.resu.(!con+!info).ask.!quer.resu.(!con+!info)

trace(Inventory) = quer.SA.UP.NB.!resu.quer. SA.UP.NB.!resu.quer.SA.UP.NB.!resu

Next, we verify whether or not two interacting web services are behavior compatible. We only need to consider writing/reading actions on internal FIFO channels between this two interacting web services. So the trace of Order service and Inventory service becomes:

trace $($ Order $)=$ !quer.resu.!quer.resu.!quer.resu

trace $($ Inventory $)=$ quer.!resuquer.!resuquer.!resu

It is evident that trace(Order) and trace(Inventory) have opposite behavior trace. So in the complete execution of Ticket Booking system, Order service and Inventory service are behavior compatible.

In fact, in an actual execution of this system, using related definition it is easy for us to verify whether or not this execution is effective or complete, whether or not two interacting web services are behavior compatible.

\section{Conclusions And Future Works}

In this paper, a novel process network approach was proposed to model dynamic behaviors of interacting context-aware web services, aiming to effectively process and take advantage of contexts and realize behavior adaptation of web services. We presented the interaction model of context-aware web services based on a formal model, context-aware process network. The context-aware process network was extended to context-aware web service network by adding a kind of sensor processes which is used to catch contextual data from external environment. Through modeling the register link's behaviors, we present how a web service can respond to its context changes dynamically. Its explicit formal behavior semantic was provided to implement context-awareness and behavior adaptation of context-aware web service. We designed a XML formatted service behavior description language named BML4WS to describe behaviors and behavior adaptation of interacting context-aware web services. Our model was demonstrated in a practical case, i.e. Ticket Booking system. The results illustrated that the proposed approach is available and flexible.

Our future work is to make an attempt to implement context-aware web service network for more web service applications. As discussed above, contextaware web service network is based on CAPN and is adequate to model service interactions in several aspects, including its parallelism and communication mechanism, compositional property, executable property and context-awareness semantics. Other formal model, such as Petri Net and process algebra, lack these advantages.

\section{ACKNOWLEDGMENTS}

This work is supported by the National Natural Science Foundation of China (Grant No.60873054, 60973013, 61073056, 61073134, 51179020), the Fundamental Research Funds for the Central Universities (Grant No.2011JC006), and Dalian Science and Technology Fund (Grant No.2010J21DW006). 


\section{REFERENCES}

[1] M. Keidl and A. Kemper, "Towards context-aware adaptable web services," in Proceedings of the 13th international World Wide Web. New York: ACM, May 2004, pp. 55-65.

[2] A. Abraham, J. Chung, and S. Han, "Web services: recent advances and applications," Journal of Digital Information Management, vol. 4, no. 1, pp. 1-3, 2006.

[3] F. Gandon and N. Sadeh, "Semantic web technologies to reconcile privacy and context awareness," Web Semantics: Science, Services and Agents on the World Wide Web, vol. 1, no. 3, pp. 241-260, 2004

[4] H. Truong and S. Dustdar, "A survey on context-aware web service systems," International Journal of Web Information Systems, vol. 5, no. 1, pp. 5-31, 2009.

[5] L. Li, D. Liu, and A. Bouguettaya, "Semantic based aspectoriented programming for context-aware web service composition," Information Systems, vol. 36, no. 3, pp. 551-564, 2011, special Issue on WISE 2009 - Web Information Systems Engineering.

[6] R. Hervas and J. Bravo, "Towards the ubiquitous visualization: Adaptive user-interfaces based on the semantic web," Interacting with Computers, vol. 23, no. 1, pp. 40-56, 2011.

[7] R. Bhatti, E. Bertino, and A. Ghafoor, "A trust-based contextaware access control model for web-services," Distributed and Parallel Databases, vol. 18, no. 1, pp. 83-105, 2005.

[8] M. Mrissa, C. Ghedira, D. Benslimane, Z. Maamar, F. Rosenberg, and S. Dustdar, "A context-based mediation approach to compose semantic web services," ACM Transactions on Internet Technology, vol. 8, no. 1, pp. 4-es, 2007.

[9] J. Simoes and S. Wahle, "The future of services in next generation networks," IEEE Potentials, vol. 30, no. 1, pp. $24-29$, jan.-feb. 2011

[10] Z. Chen, K. Ma, A. Abraham, B. Yang, and R. Sun, "An executable business model for generic web applications," in Proceedings of International Conference on Computer Information Systems and Industrial Management Applications. India: IEEE, 2010, pp. 573-577.

[11] T. Tiropanis, H. Davis, D. Millard, and M. Weal, "Semantic Technologies for Learning and Teaching in the Web 2.0 Era," IEEE Intelligent Systems, vol. 24, no. 6, pp. 49-53, 2009.

[12] L. Zhang, "Services design and optimization," IEEE Transactions on Services Computing, vol. 2, no. 2, p. 93, april-june 2009.

[13] M. Brambilla, S. Ceri, F. Facca, I. Celino, D. Cerizza, and E. Valle, "Model-driven design and development of semantic Web service applications," ACM Transactions on Internet Technology, vol. 8, no. 1, pp. 3-32, 2007

[14] I. Elgedawy, Z. Tari, and J. Thom, "Correctness-aware highlevel functional matching approaches for semantic Web services," ACM Transactions on the Web, vol. 2, no. 2, p. 12, 2008.

[15] K. Scott and R. Benlamri, "Context-aware services for smart learning spaces," IEEE Transactions on Learning Technologies, vol. 3, no. 3, pp. $214-227$, july-sept. 2010.

[16] S.-Y. Hwang, E.-P. Lim, C.-H. Lee, and C.-H. Chen, "Dynamic web service selection for reliable web service composition," IEEE Transactions on Services Computing, vol. 1, no. 2, pp. 104 -116 , april-june 2008.

[17] A. Segev and E. Toch, "Context-based matching and ranking of web services for composition," IEEE Transactions on Services Computing, vol. 2, no. 3, pp. 210 - 222, july-sept. 2009.

[18] A. Staikopoulos, O. Cliffe, R. Popescu, J. Padget, and S. Clarke, "Template-based adaptation of semantic web services with model-driven engineering," IEEE Transactions on Services Computing, vol. 3, no. 2, pp. 116 - 130, april-june 2010.

[19] D. Vieira, C. Melo, A. Bezerra, Y. Ghamri-Doudane, and N. da Fonseca, "Latincon01 - a content-oriented web cache policy under p2p video distribution systems," Latin America Transactions, IEEE (Revista IEEE America Latina), vol. 8, no. 4, pp. 349-357, aug. 2010.

[20] G. Kahn, "The semantics of a simple language for parallel programming," in Information processing, J. L. Rosenfeld, Ed. Stockholm, Sweden: North Holland, Amsterdam, Aug 1974, pp. $471-475$.

[21] H. van Dijk, H. Sips, and E. Deprettere, "Context-aware process networks," in Proceedings of IEEE International Conference on Application-Specific Systems, Architectures, and Processors. Hague: IEEE, 2003, pp. 6-16.
[22] D. Zhovtobryukh, "Context-aware Web Service Composition," Ph.D. dissertation, University of Jyvaskyla, 2006.

[23] D. Kulkarni and A. Tripathi, "A framework for programming robust context-aware applications," IEEE Transactions on Software Engineering, pp. 184-197, 2010.

[24] M. Sama, S. Elbaum, F. Raimondi, D. Rosenblum, and Z. Wang, "Context-aware adaptive applications: fault patterns and their automated identification," IEEE Transactions on Software Engineering, pp. 644-661, 2010.

[25] C. Jacob and S. Steglich, "Modeling dynamic service behavior using context functions," in Proceedings of the First International Conference on Networked Digital Technologies. Ostrava: IEEE, 2009, pp. 88-93.

[26] J. Zhou, E. Gilman, J. Palola, J. Riekki, M. Ylianttila, and J. Sun, "Context-aware pervasive service composition and its implementation," Personal and Ubiquitous Computing, vol. 15, no. 3, pp. 291-303, 2011.

[27] R. Hervás and J. Bravo, "COIVA: context-aware and ontologypowered information visualization architecture," Software: Practice and Experience, vol. 41, no. 4, pp. 403-426, 2011.

[28] A. T. Manes. (2001) Enabling open, interoperable, and smart web services - the need for shared context. [Online]. Available: http://www.w3.org/2001/03/WSWS-popa/paper29

[29] A. K. Dey, "Understanding and Using Context," Personal and Ubiquitous Computing, vol. 5(1), pp. 4-7, 2001.

[30] X. Li, Y. Fan, Q. Z. Sheng, Z. Maamar, and H. Zhu, "A petri net approach to analyzing behavioral compatibility and similarity of web services," IEEE Transactions on Systems, Man and Cybernetics, Part A: Systems and Humans, vol. 41, no. 3, pp. $510-521$, may 2011

[31] C. Yan, C. Jiang, and Q. Li, "The Composition and Analysis of Web Service Based on Petri Net," Computer Science, vol. 34, no. 2, pp. 100-103, 2007.

[32] J. Liao, H. Tan, and J. Liu, "Describing and verifying Web service using Pi-calculus," Chinese Journal of Computers, vol. 28, no. 4, pp. 635-642, 2005.

[33] X. Gu and Z. Lu, "A formal model for BPEL4WS description of web service composition," Wuhan University Journal of Natural Sciences, vol. 11, no. 5, pp. 1311-1319, 2006.

[34] H. Foster, S. Uchitel, J. Magee, and J. Kramer, "LTSA-WS: a tool for model-based verification of web service compositions and choreography," in Proceedings of the 28th International Conference on Software Engineering. Shanghai: ACM, May 2006, pp. 771774 .

[35] T. Chaari and A. Celentano, "Design of context-aware applications based on web services," INSA Lyon, France, Tech. Rep. RR-2004-033, Sep. 2004.

[36] F. L. T. Chaari and A. Celentano, "Adaptation in ContextAware Pervasive Information Systems: The SECAS Project," Pervasive Computing and Communications, pp. 400-425, 2007.

[37] Y. Dou, G. Wu, J. Xu, and X. Zhou, "A coarse-grained reconfigurable computing architecture with loop self-pipelining," Science in China Series F: Information Sciences, vol. 52, no. 4, pp. 575-587, 2009.

[38] D. Webb and A. Wendelborn, "The pagis grid application environment." Melbourne: Springer, 2003, pp. 692-693.

[39] E. De Kock, W. Smits, P. van der Wolf, J. Brunel, W. Kruijtzer, P. Lieverse, K. Vissers, and G. Essink, "YAPI: Application modeling for signal processing systems," in Proceedings of the 37th Annual Design Automation Conference. Los Angeles: ACM, 2000, pp. 402-405.

[40] M. Geilen and T. Basten, "Requirements on the execution of Kahn process networks," in Proceedings of the 12th European conference on Programming. Warsaw: Springer, 2003, pp. 319334.

[41] R. Milner, Communication and concurrency. Prentice Hall, USA, 1989.

[42] R. Cleaveland, T. Li, and S. Sims, "The concurrency workbench of the new century," User's manual, SUNY at Stony Brook, Stony Brooke, NY, USA, 2000.

[43] T. Parks, "Bounded scheduling of process networks," Ph.D. dissertation, University of California, EECS Dept., Berkeley, CA, 1995.

[44] J. Buck and E. Lee, "Scheduling dynamic dataflow graphs with bounded memory using the token flow model," in Proceedings of International Conference on Acoustics, Speech, and Signal Processing. Minneapolis: IEEE, 1993, pp. 429-432. 


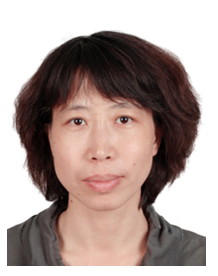

Xiuguo Zhang is an associate professor in the School of Information Science and Technology at Dalian Maritime University, Dalian, China. She received $\mathrm{PhD}$ degrees in Computer Science from Dalian Maritime University. Her research interests are Web Service, Software Engineer, etc. She has authored over $40+$ refereed journal/conference papers.

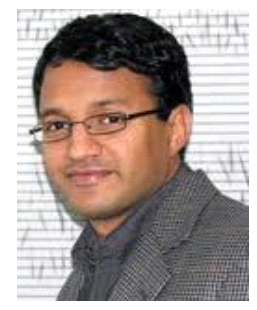

Ajith Abraham received the M.S. degree from Nanyang Technological University, Singapore, and the Ph.D. degree in Computer Science from Monash University, Melbourne, Australia. He is currently the Director of Machine Intelligence Research Labs (MIR Labs), Scientific Network for Innovation and Research Excellence, USA, which has members from more than 85 countries. He has a worldwide academic experience with formal appointments in Monash University;

Australia, Oklahoma State University, USA; Chung-Ang University, Seoul, Korea; Jinan University, Jinan, China; Rovira i Virgili University, Tarragona, Spain; Dalian Maritime University, Dalian, China; Yonsei University, Seoul, Korea; the Open University of Catalonia, Barcelona, Spain; the National Institute of Applied Sciences (INSALyon), Lyon, France; and the Norwegian University of Science and Technology (NTNU), Trondheim, Norway. He serves/has served the editorial board of over 50 International journals and has also guest edited 40 special issues on various topics. He has published more than 800 publications, and some of the works have also won best paper awards at international conferences. His research and development experience includes more than 20 years in the industry and academia. He works in a multi-discciplinary environment involving machine intelligence, network security, various aspects of networks, e-commerce, Web intelligence, Web services, computational grids, data mining, and their applications to various real-world problems. $\mathrm{He}$ has given more than 50 plenary lectures and conference tutorials in these areas.

Dr. Abraham is the Chair of IEEE Systems Man and Cybernetics Society Technical Committee on Soft Computing and a Distinguished Lecturer of IEEE Computer Society representing Europe. Dr. Abraham is a Senior Member of the IEEE, the IEEE Computer Society, the Institution of Engineering and Technology (UK) and the Institution of Engineers Australia (Australia), etc. He is the founder of several IEEE sponsored annual conferences, which are now annual events - Hybrid Intelligent Systems (HIS - 11 years); Intelligent Systems Design and Applications (ISDA - 11 years); Information Assurance and Security (IAS - 7 years); Next Generation Web Services Practices (NWeSP - 7 years), Computational Aspects of Social Networks (CASoN - 3 years), Soft Computing and Pattern Recognition (SoCPaR - 3 years), Nature and Biologically Inspired Computing (NaBIC - 3 years) are some examples. More information at: http://www.softcomputing.net

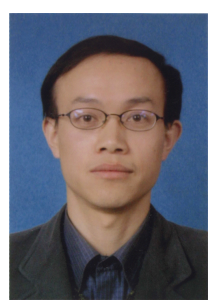

Hongbo Liu is a professor in the School of Information Science and Technology at Dalian Maritime University, Dalian, China, with an affiliate appointment in the Computer Science and Biomedical Engineering Division at Dalian University of Technology. $\mathrm{He}$ received $\mathrm{PhD}$ degrees in Computer Science from Dalian University of Technology. His research interests are in system modeling and optimization involving soft computing, nature-inspired computing, swarm intelligence, multi-agent systems, fuzzy inference systems, rough set, and probabilistic model. Application areas include neuroinformatics, cognitive computing, computational grids, web service, machine learning, data mining, etc. He has authored over $60+$ refereed journal/conference papers and book chapters and some of the works have also won best paper awards at international conferences and also received several citations. Some of the articles are available in the ScienceDirect Top 25 hottest articles. He was co-organised the China-Japan Joint Conference on Computational Geometry, Graphs and Applications (CGGA2010) at Dalian in November 2010. He is also the General Chair of the Third IEEE International Conference on Soft Computing and Pattern Recognition (SoCPaR'11) at Dalian, China in October 2011. 